\title{
Research Progress of Titanium Polypropylene Mesh in Breast Reconstruction
}

\author{
Qi Huang, Xiaohua Zhang, Lingji Guo, Yujun He* \\ Breast \& Thyroid Surgery Department, Army Military Medical University Daping Hospital, Chongqing 400042, China \\ *Corresponding author: Yujun He, huangshuan2017@163.com
}

\begin{abstract}
To investigate the application of titanium polypropylene mesh in breast reconstruction. In this study, we selected the literature data in recent 4 years to analyze the application of titanium polypropylene mesh in breast reconstruction. Using the keywords of "breast reconstruction," "titanium polypropylene mesh," "application" and "research progress," we analyzed and summarized the related research progress of titanium polypropylene mesh in breast reconstruction. The research was conducted using the analysis of titanium polypropylene mesh, titanium polypropylene mesh in breast reconstruction surgery advantages, adverse complications related to titanium polypropylene mesh in breast reconstruction surgery and preventive measures. By constantly improving these aspects in the research process, the current study has certain value, and may guide the research work of titanium mesh in breast reconstruction.
\end{abstract}

Keywords: Breast reconstruction; Titanium polypropylene mesh; Application; Research progress

Publication date: July 2021; Online publication: July 30, 2021

\section{Introduction}

As people's life quality continues to accelerate, their eating habits also change which is one of the contributing factor for the increasing incidence of breast cancer. This disease has a serious impact on women's health. At present, treatment for breast cancer is through surgery, but after surgery, the patient's body will also change. Breast reconstruction has become part of the treatment which includes implant and autologous tissue, but implant is the most common method at present. The breast reconstruction with implants is to place implants under the chest flap. However, due to the lack of tissue coverage, dilators are often used to divide the surgery into two steps. Also, the surgery method has gradually developed into delayed immediate surgical reconstruction. With the development of medical technology, ADM (acellular dermal matrix) and TCPM (titanium polypropylene mesh) were discovered which made immediate breast reconstruction is possible. Based on this, this paper mainly discusses the research progress of titanium polypropylene mesh in breast reconstruction in order to provide reference for relevant researchers.

\section{Titanium polypropylene mesh}

Titanium polypropylene mesh (TCPM) is certified by the European Union, which is safe and can be used in breast reconstruction surgery. The main structure of TCPM is nylon monofilament, which is titanized by special technology to make it histocompatibility. At the same time, TCPM also specially designed with corresponding specifications and shapes for breast reconstruction, and the commonly used specifications are $21.5 \mathrm{~cm} \times 11.5 \mathrm{~cm} \times 14 \mathrm{~cm} ; 19.5 \mathrm{~cm} \times 9.5 \mathrm{~cm} \times 12 \mathrm{~cm} ; 23.5 \mathrm{~cm} \times 13.5 \mathrm{~cm} \times 16 \mathrm{~cm}$. At the same time, TCPM also has the advantages of chemical inertia, as well as non-carcinogenic, non-reaction with human tissues, allergy-free, anti-infection and anti-tension properties. In early 2008, TCPM has been approved for application in breast reconstruction surgery, now it has been used in clinical practice for more than ten 
years.

\section{Surgery mode}

\subsection{Subcutaneous mastectomy}

Usually, the incision around the areola toward the axillary is designed to extend. For patients with benign tumor, the breast subcutaneous gland resection is directly implemented, meanwhile for patients with malignant tumor, the sentinel lymph nodes should be tracked with methylene blue and radionuclide method, and then the breast subcutaneous gland resection and sentinel lymph node biopsy should be performed. At the same time, the tissue of the nipple should be sent for examination. After biopsy confirmation that there is no tumor involved in the tissue behind the nipple, the decision should be made whether to retain the breast areola complex of the patient or otherwise.

\subsection{Immediate breast reconstruction}

The immediate breast reconstruction operation procedure involve: first, the lower edge of pectoralis major muscle of the patient is cut off, then along the pectoralis minor muscle and pectoralis major muscle, the cavity after pectoralis major muscle stripping is pointed out to the preoperative mark, then the head of operation bed is raised, the expander is placed in the cavity after pectoralis major muscle, and water is injected after adjusting the expander. After observing the shape and size of bilateral breasts, the expander was removed. According to the water injection volume of the expander, combined with the breast height, base and convex reading value measured before operation, the prosthesis with suitable volume and mesh with suitable model were selected. Then, the mesh is intermittently sutured at the lateral edge of the lower broken end of the pectoralis major muscle with absorbable suture, and the prosthesis is placed in the gap between the mesh and pectoralis major muscle. After the mesh is folded, the prosthesis is completely wrapped, and then the position of the prosthesis is adjusted to make it fully adapt to the shape of the breast. The drainage tube was placed under the flap, followed by the lower plica, and then sutured layer by layer. It is important to follow doctor's advice during perioperative period, patients can be treated with appropriate antibiotics, which can reduce the risk of infection in patients after prosthetic reconstruction surgery and all patients should take antibiotics before and within 24 hours after the operation.

\section{Application and advantages of titanium polypropylene mesh in breast reconstruction surgery}

Under normal circumstances, TCPM is mainly used to wrap the prosthesis with mesh and then put it into the subcutaneous cavity of human body or suture the mesh and the edge of pectoralis major muscle after the pectoralis major muscle is severed, and implant the prosthesis in the cavity between the mesh and pectoralis major muscle. There was no statistical difference in the surgical effect and postoperative adverse complications between the above methods.

The advantages of TCPM are as follows: (i) compared with delayed immediate breast reconstruction, the number of operations was reduced; (ii) compared with the autologous tissue flap (latissimus dorsi flap), this operation has less trauma, faster postoperative recovery and higher satisfaction; (iii) compared with biomaterials (acellular dermal matrix), it is cheaper, easier to obtain, more flexible and more stable than biomaterials. The surgery is relatively simple and less postoperative complications, and postoperative patient satisfaction is relatively high.

\section{Operation-related adverse complications and preventive measures of titanium polypropylene mesh in breast reconstruction}

At present, there are no reports of special complications of TCPM. The complications of TCPM are all related to breast reconstruction surgery. In the early postoperative period, perhaps there are some adverse 
complications, such as skin flap infection, incision cracking, hematoma and seroma, necrosis of nipple and areola, skin paresthesia and loss, incision hypertrophic scar and others. In the later stage, breast nodules, capsular contracture and mesh or prosthesis exposure may occur. Apart from that, obesity, smoking or diabetes will increase the risk factors of complications. For patients with high risk, additional precaution measure during surgery and close observation should be made after the procedure. Studies have shown that TCPM has no effect on radiotherapy, but patients with postoperative radiotherapy should be followed up regularly.

\subsection{Infection}

According to the published work, the probability of skin flap infection is about $0-6.5 \%$, and the probability of acellular dermal matrix infection is about $5.8 \%$. If the patient is severely infected, it will directly lead to the failure of surgery. Therefore, when patients have mild infection after surgery, they should take antibiotics according to the doctor's prescription which can prevent and reduce the probability of adverse complications due to infection ${ }^{[2]}$.

\subsection{Incision cracking}

The main and common adverse complication in breast reconstruction is incision dehiscence, which is directly related to the excessive tension of the flap when the prosthesis is implanted. Scientists have studied and compared TCPM assisted reconstruction with prosthesis reconstruction alone and found that there is no significant difference in the incidence of incision cracking between the two, which is not statistically significant. Therefore, the selection of suitable prosthesis can reduce the probability of incision cracking to a certain extent ${ }^{[3]}$.

\subsection{Hematoma and seroma}

It has been reported that the probability of hematoma and seroma after TCPM assisted reconstruction surgery is $3.6 \%-4.9 \%$, while the probability of hematoma and seroma after acellular dermal matrix surgery is $8.5 \%$. The application of TCPM can reduce the probability of hematoma and seroma to a certain extent [4].

\subsection{Nipple areola necrosis}

The complications of nipple and areola necrosis may occur in mastectomy with nipple and areola preserved. The main reason for this complication is that in the implementation of mastectomy, the gland behind the areola nipple was removed, the subdermal vascular network was destroyed, thus reducing the blood supply of the complex, and then the nipple areola necrosis complications occurred. It is suggested that the tissue behind the nipple should be preserved as far as possible taking into consideration the safety and permission, and the nipple compression should be avoided during the postoperative pressure bandage, which can reduce the probability of nipple areola necrosis.

\subsection{Skin paresthesia and loss}

Most of the patients with mastectomy will have skin numbness and loss after the operation, but some patients can recover by themselves during the recovery period, and some patients unable to recover by themselves. The degree of recovery varies with individual. This complication is an inevitable adverse complication of mastectomy. Studies have shown that the skin sensation of patients with immediate breast reconstruction is faster than that of patients with delayed breast reconstruction ${ }^{[5]}$. 


\subsection{Hypertrophic scar of incision}

The hypertrophic scar of incision is directly related to skin tension, skin pigment, skin part, infection, age, genetic mechanism and foreign body. It is suggested that choosing suitable prosthesis can reduce skin tension, prevent infection, and reduce the occurrence of hypertrophic scar.

\subsection{Breast nodules}

Few studies have reported that after TCPM reconstruction, small nodules will appear in the breast. In this regard, the current clinical recommendations is to take tumor resection and send for pathological examination to identify the condition of breast nodules ${ }^{[2]}$.

\subsection{Capsular contracture}

Capsular contracture is an unavoidable complication of prosthetic reconstruction and augmentation mammoplasty. The probability of capsular contracture after TCPM reconstruction is $2.0 \%-5.5 \%$ according to previous study. Because of the use of TCPM reconstruction and the follow-up period, further clinical observation and research on capsular contracture are needed. It is suggested that the risk factors should be controlled. Hairy prosthesis can reduce the probability of capsular contracture ${ }^{[6]}$. Patients with mild capsular contracture should be followed up regularly. For severe capsular contracture, the implant should be removed, and then the capsular sac should be stripped completely.

\subsection{Exposure of mesh or prosthesis}

In general, the exposure of TCPM or prosthesis can be caused by incision dehiscence or whip necrosis. It is suggested that the prosthesis can be directly removed, and then the operation can be finished or delayed. At the same time, try to use the breast intercostal perforator flap to repair, do not need to remove the implanted prosthesis, this can also achieve better clinical effect ${ }^{[7]}$.

\section{Conclusion}

To sum up, the key problem of implant breast reconstruction is the amount of tissue coverage. In recent years, the commonly used operations are nipple conserving mastectomy and skin conserving mastectomy, which can provide sufficient skin tissue for breast reconstruction, but the lack of muscle tissue increases the operation difficulty to a certain extent. TCPM is an alternative material to increase muscle coverage, which can simplify the surgery and will not increase the postoperative adverse complications. In the postoperative process, patients' satisfaction with the operation is relatively high, and it has been widely used in Europe. In China, however, the application of breast reconstruction is relatively low. The implementation of breast reconstruction using TCPM is simple and highly safe; therefore, it is recommended for clinical application in breast reconstruction.

\section{Disclosure statement}

The authors declare that there is no conflict of interest.

\section{References}

[1] Ishiba T, Aruga T, Miyamoto H, et al., 2021, Short- and Long-Term Outcomes of Immediate Breast Reconstruction Surgery after Neoadjuvant Chemotherapy. Surgery Today, Online ahead of print.

[2] Atiyeh BS, Chahine F, 2021, Letter to the Editor: Sub-muscular Reconstruction after NAC Sparing Mastectomy-Direct to Implant Breast Reconstruction with Human ADM versus Tissue Expander. Aesthetic Plastic Surgery, 45(3): 1370-1371. 
[3] Sun J, Ge W, Liu M, 2021, Comparison of the Effect of Xenogeneic Acellular Dermal Matrix and New Titanium Mesh in Immediate Breast Reconstruction after Breast Cancer Resection. Clinical Medicine, 41(2): 34-35.

[4] Li T, Shi J, 2021, Clinical Application of Titanium Polypropylene Mesh in Breast Prosthesis Reconstruction after Tissue Expansion. Journal of Nanjing Medical University (Natural Science Edition), 41(2): 238-243.

[5] Yang Q, Chen W, Chen S, et al., 2019, Application of Titanium Polypropylene Mesh in Immediate Breast Reconstruction after Breast Cancer Surgery with Silicone Gel Prosthesis. Chinese Journal of General Surgery, 28(11): 1393-1399.

[6] Sgarzani R, Pasquali S, Buggi F, 2021, Invited Response on: "Submuscular Reconstruction After NAC Sparing Mastectomy: Direct to Implant Breast Reconstruction with Human ADM Versus Tissue Expander". Aesthetic Plastic Surgery, 45(3): 1372-1374.

[7] Wu Y, Shi J, Liu X, et al., 2018, Clinical Application of Titanium Polypropylene Mesh in Immediate Breast Reconstruction. Chinese Journal of Cancer, 28(4): 297-302. 